\title{
Dynamics of the plume produced by nanosecond ultraviolet laser ablation of metals
}

\author{
B. Toftmann and J. Schou* \\ Department of Optics and Fluid Dynamics, Risф National Laboratory, DK-4000 Roskilde, Denmark \\ J. G. Lunney \\ Physics Department, Trinity College, Dublin 2, Ireland \\ (Received 6 November 2002; published 4 March 2003)
}

\begin{abstract}
The dynamics of the ablation plume of a partially ionized plasma produced by a nanosecond UV laser with different irradiation spot geometries has been explored. We have used an ensemble of quartz crystal microbalances to make the first systematic and quantitative study of how the shape of the plume varies as the aspect ratio $(b / a)$ of the elliptical laser spot is varied by about a factor of ten. The flip-over effect can be described by the adiabatic expansion model of Anisimov et al. using a value of the adiabatic constant of about $\gamma$ $=1.4$. We have also studied the forward peaking of the ablation plume for a large number of metals at the same laser fluence. Contrary to earlier reports, we find that the more refractory metals have the broader angular distributions.
\end{abstract}

DOI: 10.1103/PhysRevB.67.104101

PACS number(s): 79.20.Ds

\section{INTRODUCTION}

The interaction of a pulsed-laser beam with a solid leads to a complicated sequence of processes. ${ }^{1,2}$ High intensity ns laser pulses have been used both for fundamental and applied studies for more than two decades, but many of the basic phenomena occurring during laser irradiation of solids are not yet fully understood. The formation and expansion of a plume produced by laser beam irradiation of a surface is the starting point for studies of plume dynamics, ${ }^{3-8}$ film production by pulsed laser deposition, ${ }^{9-12}$ and microanalysis. ${ }^{13}$ Surprisingly, there is no complete theoretical treatment of a time-limited expansion process, even for a neutral expanding plume. ${ }^{4,14-18}$

Generally, the interaction of a ns laser beam with a target can be described as a three-stage process: (i) effectively instantaneous conversion of absorbed laser light to heat, (ii) evaporation, and possibly strong explosive ejection during the later part of the laser pulse leading to the generation of a dense plume of vapor with atoms and clusters and plasma, and (iii) finally a three-dimensional expansion of the plume. Essentially, the first two stages can be considered as one dimensional. For an irradiated area of the order of $1 \mathrm{~mm}^{2}$ the dense plume is comprised of a layer which may be about 100 $\mu \mathrm{m}$ thick at the end of the pulse depending on the laser fluence. The two first stages are partly simultaneous, since the laser heating of the solid through the initially generated plume continues until the end of the laser pulse except at very high intensities, for which the plume becomes opaque. ${ }^{7}$ For ns ablation at comparatively low intensities a significant fraction of the laser energy can be absorbed by the plume. Even at intensities close to the threshold for ablation $(0.2-$ $1.0 \mathrm{GW} / \mathrm{cm}^{2}$ ) a considerable part of the plume is ionized. ${ }^{19-21}$ After the end of the laser pulse there is little further transfer of energy or material to the ablation plume. The subsequent adiabatic expansion of the ablation plume in stage (iii) is largely determined by the shape of the plume at the end of the laser pulse. ${ }^{14,16,17,22}$

The initial formation of the plume in stage (i) and (ii) is a complex process, which may involve vaporization and phase explosion. ${ }^{23-25}$ For the emission of neutrals during ablation the evolution of the plume can be characterized by the formation of a Knudsen layer, ${ }^{4}$ over which the velocity distributions change from a half-space to a full three-dimensional distribution. In the present work we base our analysis of the dynamics of the plume on the geometrical shape rather than on the internal composition or the internal distribution of the plume particles.

\section{PLUME DYNAMICS}

Since at the end of the laser pulse irradiation the lateral dimensions exceed the thickness of the plume by several orders of magnitude, the higher value of the pressure gradient along the surface normal drives the plume motion primarily in forward direction. The pressure gradients parallel to the surface are much smaller and lead to a correspondingly slower plume expansion in lateral direction. Actually there are three plume characteristics reflecting the initial shape of the plasma plume. ${ }^{10,16,17}$ These are (a) the strongly peaked angular distribution of ablated atoms perpendicular to the surface, (b) the flip-over effect of the plume, and (c) the broadening of the angular distribution with decreasing laser beam spot. The first effect, the forward peaked distribution, is well known. ${ }^{5,8,10}$ The flip-over effect occurs when the plume produced by an elliptical or rectangular laser beam spot evolves in such a way during expansion that the major axis of the expanded plume lies at $90^{\circ}$ to that of the initial spot. This effect is particularly distinct for a laser spot which is very narrow in one direction. This lateral expansion of the plume can also be explained on the basis of the initial pressure gradients. ${ }^{17,22}$ The common origin of these phenomena is a key-point for the analysis of the plume expansion. ${ }^{10}$ The present work is a combined study of the two first effects (a) the width of the peaked angular distribution and (b) the flipover effect. The effect of the size of the laser beam spot on the width of the angular distribution (c) has been studied thoroughly by other groups. ${ }^{10,14,26,27}$ 


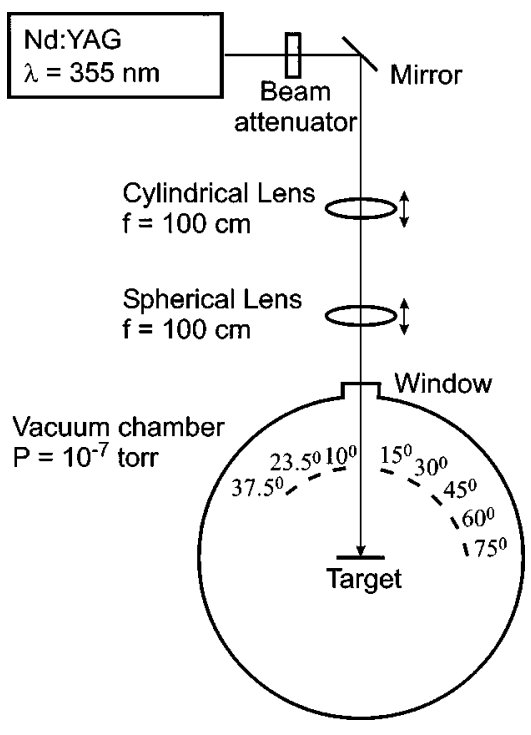

FIG. 1. The experimental setup.

The flip-over effect has been observed by several groups, but the number of data points is rather limited. ${ }^{28-32}$ In addition, some studies were complicated by the use of compound target materials, such as high- $T_{c}$ superconductors, or the presence of a background gas. ${ }^{10,22,33,34}$ Also a number of studies of the forward peaked angular distribution have been carried out. ${ }^{5,8,10,27,35}$ Surprisingly, there are practically no data for the angular distribution for a series of metals irradiated by UV lasers under the same experimental conditions.

We have studied the flip-over effect for the simplest case, a one-component metal target in vacuum, and for the first time made a systematic, quantitative analysis of the flip-over effect. In addition, we have measured the angular distribution of the ablated atoms for a wide selection of metals including both volatile and refractory elemental targets for UV laser irradiation under the same experimental conditions.

We have analyzed the results in terms of two existing models for an adiabatic expansion by Anisimov et al. ${ }^{16,22}$ and Singh and Narayan. ${ }^{17}$ While the equations of motion for the plume are similar in the two models, the latter authors have assumed that the spatial temperature gradients are zero, even though there is no mechanism of sustaining a finite temperature at the outer edge of the plume during the free expansion. ${ }^{17}$ The model of Anisimov et al. does not have this limitation and treats the plume as an expanding self-similar cloud. This model was developed to describe the expansion of neutral gas, but we have obtained good agreement with our data for the ionic component of a laser ablation plume. $^{21,36}$ Here we are using measurements of the deposition, due to neutrals and ions, to find the shape of the plume expansion, and these measurements are compared with the theoretical values from Anisimov et $a l^{22}$

\section{EXPERIMENT}

The ablation was performed with a frequency-tripled $\mathrm{Nd}$ :YAG laser at $355 \mathrm{~nm}$ directed at normal incidence onto a metal target in a vacuum of $10^{-7} \mathrm{mbar}$, in the same target chamber geometry as used earlier (Fig. 1). 19,36,37 The duration of the laser pulse was $6 \mathrm{~ns}$ and the fluence was $2.0 \mathrm{~J} / \mathrm{cm}^{2}$ corresponding to an intensity of $3.3 \times 10^{8} \mathrm{~W} / \mathrm{cm}^{2}$. The area of the laser beam spot was $0.041 \pm 0.005 \mathrm{~cm}^{2}$, but the shape was changed during the measurements of the flip-over effect. The size and shape of the beam spot were evaluated from photographs of the laser footprint on the target.

The angular distribution of the ablated flux was measured in situ $80 \mathrm{~mm}$ from the target using a circular array of eight quartz crystal microbalances (QCM's) in the horizontal plane as shown in Fig. 1. The crystals were located at $10^{\circ}, 15^{\circ}$, $23.5^{\circ}, 30^{\circ}, 37.5^{\circ}, 45^{\circ}, 60^{\circ}$, and $75^{\circ}$ with respect to the surface normal, and each crystal had a $6 \mathrm{~mm}$ diameter active area. Each run was taken on a fresh target spot, typically with 200-400 pulses with a repetition frequency of $0.1 \mathrm{~Hz}$ and with a subsequent relaxation time for the crystals of 4-6 h. For a silver target the deposition rate on the crystal electrodes closest to the normal corresponds to 5 $\times 10^{13} \mathrm{Ag}$ atoms $/\left(\mathrm{cm}^{2}\right.$ pulse $)$.

We also measured the weight loss per pulse (1.96 $\pm 0.16) \times 10^{15} \mathrm{Ag}$ atoms/pulse at $2 \mathrm{~J} / \mathrm{cm}^{2}$, by weighing the target before and after a number of laser pulses on a standard laboratory balance.

\section{RESULTS AND DISCUSSION}

\section{A. The flip-over effect}

The flip-over effect was studied by changing the ellipticity of the beam spot (in the $X-Y$ plane) with a spherical and a cylindrical lens placed in different distances from the target. Since the area, and thus the fluence, were kept constant, the only varying quantity is the ratio $b / a$ of the axes of the ellipse (with $a$ being one-half of the diameter along the $X$ axis and the quartz crystal microbalances located in the $X-Z$ plane). Figure 2 shows the angular distributions for values of the aspect ratio $b / a$ over almost one order of magnitude. The data have been fitted with the angular distribution $F(\theta)$ from Anisimov's model ${ }^{21,36}$

$$
F(\theta) / F(0)=\left(1+\tan ^{2} \theta\right)^{3 / 2}\left[1+\left(Z_{\mathrm{inf}} / X_{\mathrm{inf}}\right)^{2} \tan ^{2} \theta\right]^{-3 / 2},
$$

where the fitting parameter $Z_{\text {inf }} / X_{\text {inf }}$ is the ratio of the limiting value of the cloud front along the $Z$ axis (in forward direction into vacuum) and the value of the front in horizontal direction along the $X$ axis. This distribution accounts for the deposition per unit area on a hemisphere in contrast to the expressions indicated in Ref. 16 which have been derived for deposition on a planar substrate. According to Ref. 16 the value of the distribution $F(\theta)$ for $\theta=0$ is

$$
F(0)=Y\left(Z_{\text {inf }} / X_{\text {inf }}\right)^{2} /\left(2 \pi d^{2}\right),
$$

where $Y$ is the total number of ablated atoms per pulse and $d$ the distance from the target to a (hemispherical) substrate surface.

The angular distribution $F(\theta)$ of ablated atoms or ions is often expressed as

$$
F(\theta) / F(0)=\cos ^{p} \theta+B \cos \theta
$$




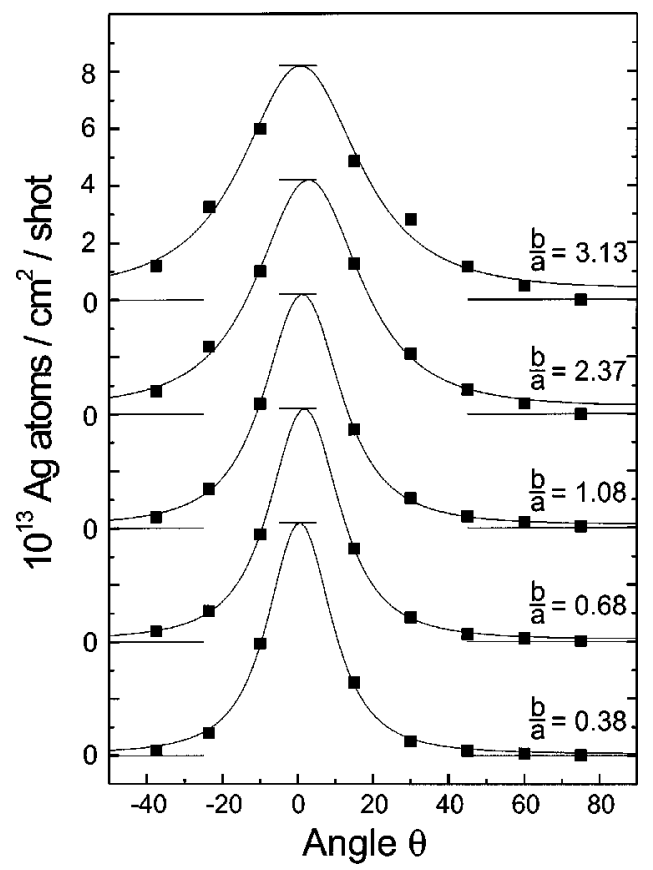

FIG. 2. The absolute deposition rate as a function of angle for $2.0 \mathrm{~J} / \mathrm{cm}^{2}$ at $355 \mathrm{~nm}$. Fits [Eq. (1)] based on Anisimov's model are shown.

where $p$ is an exponent and $B$ a constant. The extra term $B \cos \theta$ has been added to account for a broad background. ${ }^{38}$ For $B=0, p$ typically varies between 5 and $15 .^{10,19,27,39}$ This expression is essentially an empirical approximation in contrast to Eq. (1) employed by us. The horizontal angular distribution curves in Fig. 2 clearly demonstrate the flip-over effect; there is a narrower (full width at half maximum $\Delta \theta$ $=20^{\circ}$ ) horizontal angular distribution for elliptical spots with the longer dimension horizontal $(b / a=0.4)$ and a broader $\left(\Delta \theta=36^{\circ}\right)$ distribution for spots with the longer dimension vertical $(b / a=3)$. This full width at half maximum $\Delta \theta$ can immediately be obtained from Eq. (1):

$$
\Delta \theta=2 \arctan \left[\left(2^{2 / 3}-1\right) /\left(Z_{\mathrm{inf}}^{2} / X_{\mathrm{inf}}^{2}-2^{2 / 3}\right)\right]^{1 / 2},
$$

when the fitting parameter $Z_{\text {inf }} / X_{\text {inf }}$ has been determined. The width $\Delta \theta$ is shown as a function of the ratio $b / a$ in Fig. 3. Even though the flip-over effect is not new, ${ }^{17,34}$ our data set is the most systematic one to date.

We have plotted the fitting parameters from Fig. 3 as a function of the aspect ratio $b / a$ and included a set of curves calculated from Anisimov's theory ${ }^{22}$ in Fig. 4. For each value of the adiabatic constant $\gamma$ we have determined $Z_{0}$, the initial value of the cloud thickness before the expansion, for a circular beam spot $(b / a=1)$, and assumed that this value of $Z_{0}$ is valid for all aspect ratios $(b / a)$. The curves have then been normalized to the experimentally obtained average value of $Z_{\text {inf }} / X_{\text {inf }}$ at $b / a=1$. The best fit with a power function to the experimental points gives a value $\gamma=1.42$ \pm 0.07 .

The total mass loss $(1.96 \pm 0.16) \times 10^{15} \mathrm{Ag}$ atoms/pulse at $2 \mathrm{~J} / \mathrm{cm}^{2}$, is constant over the range of values of $b / a$ (0.4 to 3). The normal incidence of the laser beam means that the

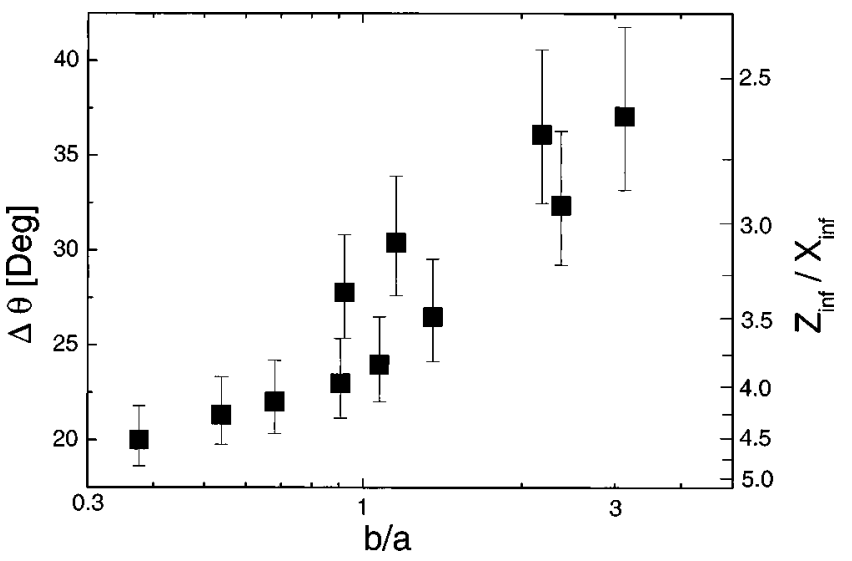

FIG. 3. The variation of the full width at half maximum $\Delta \theta$ for the angular distributions shown as a function of the ratio of the elliptic axes $(b / a) . \Delta \theta$ has been determined by Eq. (4) from the asymptotic aspect ratio $Z_{\text {inf }} / X_{\text {inf }}$ found from the fits in Fig. 2. The value of this aspect ratio is indicated on the right-hand axis.

laser plume is symmetrical with respect to the $Z$ axis, so that the angular distribution can be integrated numerically over the full hemisphere. This gives a total number of ablated particles $Y=2.60 \times 10^{15} \mathrm{Ag}$ atoms/pulse.

The scatter of the data points is primarily caused by the difficulty of measuring precisely the values of the major and minor axes of the beam spot. Sputtering of the deposited silver film may also lead to a discrepancy between the total number of ablated atoms calculated from the deposition rate and that measured directly by weight loss. This effect would primarily occur at angles close to the normal, where the ablated ions have the largest energy. ${ }^{3,37}$ Obviously, we are not able to measure the deposition rate at $\theta=0^{\circ}$ because of the laser beam, and the value of the angular distribution in this direction $F(0)$, Eq. (1), is found from those fits which give the best overall agreement with all data points in Fig. 2. A

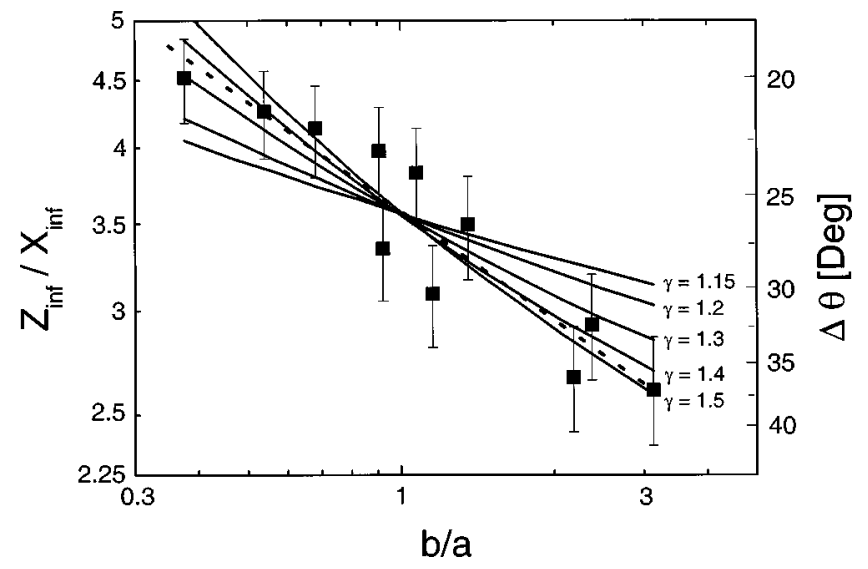

FIG. 4. The data points show the asymptotic aspect ratio $Z_{\text {inf }} / X_{\text {inf }}$ from Fig. 3 as a function of the aspect ratio $(b / a)$ in a logarithmic plot. The corresponding value of $\Delta \theta$ is shown on the right-hand axis. The solid lines for $Z_{\text {inf }} / X_{\text {inf }}$ are calculated from Anisimov et al. (Ref. 22). The curves have been normalized to the average value of $Z_{\mathrm{inf}} / X_{\mathrm{inf}}$ at $b / a=1$. The dashed line is the best fit corresponding to $\gamma=1.42$. 
significant sputtering of the deposit would mean that the true value of the deposition rate $F(0)$ should have been increased relatively to what we have shown in Fig. 2. The work by Fähler $e t a l .{ }^{20}$ shows that the deposition rate for silver at 2.0 $\mathrm{J} / \mathrm{cm}^{2}$ can be influenced by sputtering, but also that the reduction does not exceed a factor of 0.2. We have, therefore, not attempted to perform any corrections of the data, but used the data points directly with a minimum of assumptions in Figs. 3 and 4. If the true deposition rate along the normal were a factor of 1.2 higher than estimated in the present work, the plume would be narrower. However, this change would only reduce the width $\Delta \theta$ with few degrees, and the overall tendency would be similar to that showed in Figs. 3 and 4.

Anisimov's model ${ }^{22}$ can be used to calculate the total number of ablated particles $Y$ from Eq. (2), since $F(0)$ $\left(=8.2 \times 10^{15} \mathrm{Ag}\right.$ atoms $\left./ \mathrm{cm}^{2}\right)$ and $Z_{\text {inf }} / X_{\text {inf }}(=3.56)$ are known from the distributions in Fig. 2. This number, $Y$ $=2.6 \times 10^{15} \mathrm{Ag}$ atoms/pulse, is identical to the numerically integrated result without any assumption of the value of the adiabatic constant $\gamma$. Our data in Figs. 3 and 4 correspond to a value of $\gamma=1.42 \pm 0.07$. This is less than the value for a (neutral) monatomic gas $\left(\gamma=\frac{5}{3}\right)$, but also somewhat more than the value obtained by us for the ionic component of a partly ionized plasma $(\gamma \cong 1.25)$ (Ref. 36) and that for a plasma indicated by Zel'dovich and Raizer $(\gamma=1.24) .{ }^{40}$

\section{B. The angular distribution of different metals}

We have measured the angular distribution of deposition for a number of metals covering a wide range of volatility and atomic mass. All these measurements were made with a circular laser beam spot and a fluence of $2 \mathrm{~J} / \mathrm{cm}^{2}$. We have reported previously in Ref. 21 that the total ablation yield is strongly dependent on the volatility. In Fig. 5 we have plotted the width $\Delta \theta$ of the angular distribution as a function of the ablated yield. There is a clear trend showing the width of the distribution to be narrower for the more volatile metals.

The width of peak of the angular distribution about the normal has been a controversial point in the literature..$^{10,38}$ Some authors have suggested that the angular distribution becomes increasingly narrow with the atomic mass, ${ }^{38,41}$ whereas Akhsakhalyan et al. ${ }^{42}$ have suggested that volatile materials exhibited a broader distribution than refractory ones. The latter measurements ${ }^{38,41,42}$ were performed with the laser wavelength of $1064 \mathrm{~nm}$ and at a much higher fluence than considered here. The Anisimov model ${ }^{16}$ predicts that, for a given spot, smaller values of the initial cloud thickness $Z_{0}$ lead to narrower angular distributions. For each value of $Z_{\text {inf }} / X_{\text {inf }}$ and $\gamma$ in Fig. 5 one can unambiguously assign a value of the initial plume ratio $\left(Z_{0} / X_{0}\right)=(c / a)$, where $c$ is the distance of the plume front from the surface at the end of the laser pulse and $a$ the radius of the beam spot. ${ }^{16}$

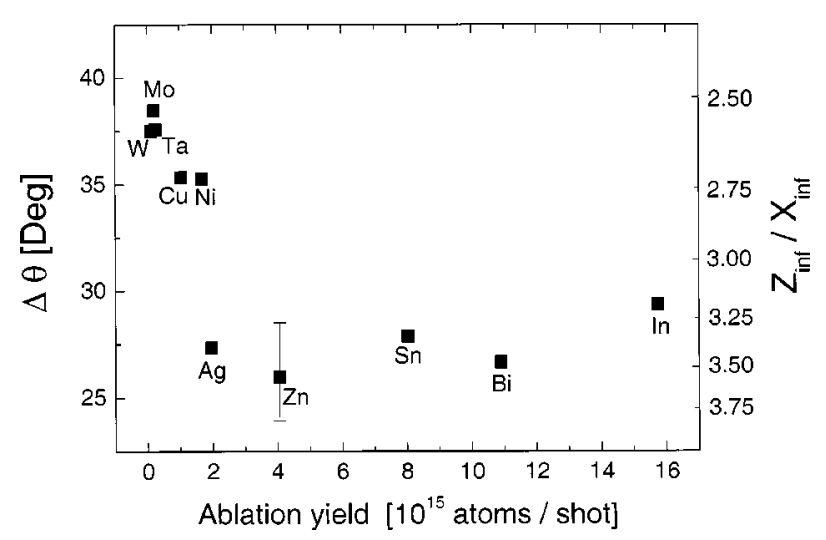

FIG. 5. The full width at half maximum $\Delta \theta$ from Eq. (4) for the angular distributions as a function of the total ablation yield per pulse (from Ref. 21) for a laser fluence of $2 \mathrm{~J} / \mathrm{cm}^{2}$. The laser beam spot was circular. The fits are based on Eq. (1). The corresponding value of $Z_{\text {inf }} / X_{\text {inf }}$ is marked on the right-hand axis. A representative error bar is also shown.

Thus, our results suggest that the more volatile metals have the smaller values of the initial cloud thickness $Z_{0}$. This can be understood by noting that in the case of a volatile metal, the energy absorbed in the ablated material is distributed among a larger number of particles, and the expansion velocity will be correspondingly lower, leading to thinner vapor cloud at the end of the pulse. With the value $\gamma=1.42$ we obtain a value for $c$ of $120 \mu \mathrm{m}$ for the refractory metals and $50 \mu \mathrm{m}$ for the volatile ones.

\section{CONCLUSION}

In summary, we have explored the shape of the plume in ns laser ablation of metals for different laser beam spot geometries. We have made the first systematic, quantitative study of the lateral flip-over effect for the simple case of a metal plume in vacuum. The width of the angular distribution increased by a factor of 2 as the aspect ratio of the elliptic beam spot axes $(b / a)$ was varied from 0.4 to 3 . The flip-over effect can be described by the adiabatic expansion model of Anisimov et al. using a value $\gamma=1.42 \pm 0.07$. In contrast to earlier measurements, it was found that refractory metals show broader angular distributions than more volatile metals. Generally, the expansion of the ablation plume is well described by Anisimov's model using realistic values of the expansion parameters.

\section{ACKNOWLEDGMENTS}

The work has been supported by the EU Growth Program under Contract No. G6RD-CT1999-00084, a grant from the Danish Natural Science Research Council and a grant from Enterprise Ireland.

\footnotetext{
*Email address: j.schou@ risoe.dk

${ }^{1} \mathrm{M}$. von Allmen and Andreas Blatter, Laser-Beam Interactions with Materials: Physical Principles and Applications (Springer, Berlin, 1995).
}

${ }^{2}$ Laser Ablation and Desorption, edited by J. C. Miller and R. F. Haglund, Experimental Methods in the Physical Sciences Vol. 30 (Academic Press, New York, 1998).

${ }^{3}$ B. Toftmann, J. Schou, T. N. Hansen, and J. G. Lunney, Phys. 
Rev. Lett. 84, 3998 (2000).

${ }^{4}$ D. Sibold and H. M. Urbassek, Phys. Rev. A 43, 6722 (1991).

${ }^{5}$ S. Amoruso, R. Bruzzese, N. Spinelli, and R. Velotta, J. Phys. B 32, R131 (1999).

${ }^{6}$ S. I. Anisimov and B. S. Luk'yanchuk, Phys. Usp. 45, 293 (2002).

${ }^{7}$ C. R. Phipps and R. W. Dreyfus, in Laser Ionization Mass Analysis, edited by A. Vertes, R. Gijbels, and F. Adams, Chemical Analysis Series No. 124 (Wiley, New York, 1993), p. 369.

${ }^{8}$ K. L. Saenger, in Pulsed Laser Deposition of Thin Films, edited by D. B. Chrisey and G. B. Hubler (Wiley-Interscience, New York, 1994), p. 199.

${ }^{9}$ D. H. Lowndes, D. B. Geohegan, A. A. Puretzky, D. P. Norton, and C. M. Rouleau, Science 273, 898 (1996).

${ }^{10}$ D. H. Lowndes, in Laser Ablation and Desorption (Ref. 2), p. 475.

${ }^{11}$ P. R. Willmott and J. R. Huber, Rev. Mod. Phys. 72, 315 (2000).

${ }^{12}$ D. Bäuerle, Laser Processing and Chemistry, 3rd ed. (Springer, Berlin, 2000).

${ }^{13}$ R. E. Russo and X. Mao, in Laser Ablation and Desorption (Ref. 2), p. 375 .

${ }^{14}$ D. Sibold and H. M. Urbassek, J. Appl. Phys. 73, 8544 (1993).

${ }^{15}$ R. Kelly and R. W. Dreyfus, Surf. Sci. 198, 263 (1988).

${ }^{16}$ S. I. Anisimov, D. Bäuerle, and B. S. Luk'yanchuk, Phys. Rev. B 48, 12076 (1993).

${ }^{17}$ R. K. Singh and J. Narayan, Phys. Rev. B 41, 8843 (1990).

${ }^{18}$ A. Peterlongo, A. Miotello, and R. Kelly, Phys. Rev. E 50, 4716 (1994).

${ }^{19}$ T. N. Hansen, J. Schou, and J. G. Lunney, Europhys. Lett. 40, 441 (1997).

${ }^{20}$ S. Fähler, K. Sturm, and H.-U. Krebs, Appl. Phys. Lett. 75, 3766 (1999).

${ }^{21}$ B. Thestrup, B. Toftmann, J. Schou, B. Doggett, and J. G. Lunney, Appl. Surf. Sci. 197-198, 175 (2002).

${ }^{22}$ S. I. Anisimov, B. S. Luk'yanchuk, and A. Luches, Appl. Surf. Sci. 96-98, 24 (1996).

${ }^{23}$ R. Kelly and A. Miotello, J. Appl. Phys. 87, 3177 (2000).
${ }^{24}$ X. Zhang, S. S. Chu, J. R. Ho, and C. P. Grigoropoulus, Appl. Phys. A: Mater. Sci. Process. 64, 545 (1997).

${ }^{25}$ J. H. Yoo, S. H. Jeong, R. Greif, and R. E. Russo, J. Appl. Phys. 88, 1638 (2000).

${ }^{26}$ M. Eyett and D. Bäuerle, Appl. Phys. Lett. 51, 2054 (1987).

${ }^{27}$ I. Weaver and C. L. S. Lewis, J. Appl. Phys. 79, 7216 (1996).

${ }^{28}$ R. Kelly, A. Miotello, A. Mele, and A. Giardini Giudoni, in Laser Ablation and Desorption (Ref. 2), p. 225.

${ }^{29}$ A. Mele, A. Giardini Guidoni, R. Kelly, A. Miotello, S. Orlando, R. Teghil, and C. Flamini, Nucl. Instrum. Methods Phys. Res. B 116, 257 (1996).

${ }^{30}$ C. N. Afonso, R. Serna, F. Catalina, and D. Bermejo, Appl. Surf. Sci. 46, 249 (1990).

${ }^{31}$ F. Antoni, C. Fuchs, and E. Fogarassy, Appl. Surf. Sci. 96-98, 50 (1996).

${ }^{32}$ H.-U. Krebs, S. Fähler, and O. Bremert, Appl. Surf. Sci. 86, 86 (1995).

${ }^{33}$ R. E. Muenchausen, K. M. Hubbard, S. Foltyn, R. C. Estler, and N. S. Nogar, Appl. Phys. Lett. 56, 578 (1990).

${ }^{34}$ R. K. Singh, N. Biunno, and J. Narayan, Appl. Phys. Lett. 53, 1013 (1988).

${ }^{35}$ H. Dang and Q. Qin, Phys. Rev. B 60, 11187 (2000).

${ }^{36}$ T. N. Hansen, B. Toftmann, J. Schou, and J. G. Lunney, Appl. Phys. A: Mater. Sci. Process. 69, S601 (1999).

${ }^{37}$ T. N. Hansen, J. Schou, and J. G. Lunney, Appl. Phys. Lett. 72, 1829 (1998).

${ }^{38}$ E. Buttini, A. Thum-Jäger, and K. Rohr, J. Phys. D 31, 2165 (1998).

${ }^{39}$ W. Svendsen, J. Schou, T. N. Hansen, and O. Ellegaard, Appl. Phys. A: Mater. Sci. Process. 66, 494 (1998).

${ }^{40}$ Ya. R. Zel'dovich and Yu. P. Raizer, Physics of Shock Waves and High-Temperature Hydrodynamic Phenomena (Academic, New York, 1966), p. 209.

${ }^{41}$ L. Torrisi, L. Ando, G. Ciavola, S. Gammino, and A. Barna, Rev. Sci. Instrum. 72, 68 (2001).

${ }^{42}$ A. D. Akhsakhalyan, S. V. Gaponov, V. I. Luchin, and A. P. Chirimanov, Sov. Phys. Tech. Phys. 33, 1146 (1988). 